\title{
Possible Tetraquark Explanation for the Proposed Zcs(3985)-
}

Joseph Bevelacqua

Funding: The author(s) received no specific funding for this work.

Potential competing interests: The author(s) declared that no potential competing interests exist.

\section{Abstract}

The recently proposed $\mathrm{Z}_{\mathrm{CS}}(3985)^{-}$structure is investigated using a first-order tetraquark mass formula. This mass relationship is based on weakly bound $D_{s}{ }^{-}$(c-bar s) $+D^{* 0}(2007)\left(c\right.$ u-bar) and $D_{s}{ }^{*}(c-b a r ~ s)+D^{0}(c u-$ bar) meson clusters and provides a reasonable prediction (within about 3\%) of the measured $Z_{c s}(3985)^{-}$ mass.

\subsection{Introduction}

The BESIII Collaboration ${ }^{1}$ recently reported the first candidate for a charged hidden-charm tetraquark with strangeness that decays into $\mathrm{D}_{\mathrm{s}}{ }^{-}$(c-bar s) $+\mathrm{D}^{* 0}(2007)\left(\mathrm{c} \mathrm{u}\right.$-bar) and $\mathrm{D}_{\mathrm{s}}{ }^{*}$ (c-bar s) $+\mathrm{D}^{0}$ (c u-bar). The proposed $\mathrm{Z}_{\mathrm{Cs}}(3985)^{-}$tetraquark is assigned a spin and parity of $1^{+}$with a resonance mass of $3985.2 \mathrm{MeV} / \mathrm{c}^{2}$ at $5.3 \sigma$ over the pure contributions from the conventional charmed mesons ${ }^{1}$.

The $\mathrm{Z}_{\mathrm{CS}}(3985)^{-}$tetraquark candidate would couple to at lease one of the following structures ${ }^{1}: \mathrm{D}_{\mathrm{S}}{ }^{-}+$ $D^{* 0}(2007)$ and $D_{S}{ }^{*}+D^{0}$. It has unit charge with a most likely c c-bar s u-bar quark configuration. As such it would become the first observed $Z_{\mathrm{cs}}$ tetraquark ${ }^{1}$.

In this paper, the first-order tetraquark mass formula of Refs. 2 - 7 is applied to evaluate the possible configuration of the $Z_{C S}(3985)^{-}$state. A guide to the possible tetraquark configuration is provided in Ref. 1 that suggests possible $D_{S}{ }^{-}+D^{* 0}(2007)$ and $D_{S}{ }^{*-}+D^{0}$ configurations. These configurations are investigated assuming a c c-bar s u-bar quark structure and the first-order tetraquark mass formula ${ }^{2-7}$.

\subsection{Model and Formulation}

Zel'dovich and Sakharov ${ }^{8,9}$ proposed a semiempirical mass formula that provides a prediction of mesons and baryons in terms of effective quark masses. Within this formulation, quark wave functions are assumed to reside in their lowest $1 \mathrm{~S}$ state. These mass formulas are used as the basis for deriving a first-order tetraquark mass formula. In particular, the model proposed in this paper assumes the tetraquark is partitioned into two clusters with the interaction between the clusters providing a minimal contribution to the tetraquark mass.

The meson mass (M) formula of Refs. 2 and 3 is: 


\section{$M_{m}=\delta_{m}+m_{1}+m_{2}+b_{m}\left[m_{0}^{2} /\left(m_{1} m_{2}\right)\right] \sigma_{1} \cdot \sigma_{2}$}

where $m_{1}\left(m_{2}\right)$ are the mass of the first (second) quark comprising the meson, $m_{0}$ is the average mass of a first generation quark ${ }^{8,9}$, and the $\sigma_{i}(i=1$ and 2$)$ are the spin vectors for the quarks incorporated into the meson. The parameters $\delta_{m}$ and $b_{m}$ are $40 \mathrm{MeV} / \mathrm{c}^{2}$ and $615 \mathrm{MeV} / \mathrm{c}^{2}$, respectively ${ }^{9}$.

The last term in Eq. 1 represents the spin-spin interaction of the quarks and $\sigma_{1} \cdot \sigma_{2}$ is the scalar product of the quark spin vectors. $\boldsymbol{\sigma}_{\mathbf{1}} \cdot \boldsymbol{\sigma}_{\mathbf{2}}$ has the value $-3 / 4,+1 / 4$ for pseudoscalar and vector mesons, respectively ${ }^{9}$.

In formulating the tetraquark mass formula, effective quark masses provided by Griffiths ${ }^{10}$ are utilized. These effective masses for $d, u, s, c, b$, and t quarks are 340, 336, 486, 1550, 4730, and $177000 \mathrm{MeV} / \mathrm{c}^{2}$, respectively. These masses are utilized in Eq. 1.

These six quarks are arranged in three generations: $[d(-1 / 3), u(+2 / 3)],[s(-1 / 3), c(+2 / 3)]$, and $[b(-1 / 3)$, $t(+2 / 3)]^{11}$. The three generations are specified by the square brackets and the quark charges are given within parentheses.

\subsection{First-Order Mass Formula for the $Z_{c s}(3985)^{-}$}

The spin of a tetraquark within the first order mass formula is determined by coupling the two meson clusters

$J^{\pi}=J^{\pi}(1) \times L \times J^{\pi}(2)$

where the first-order mass formula assumes a minimally interacting $L=0$ configuration ${ }^{2,3}$ between the meson clusters. The $J^{\pi}=1^{+}$assignment ${ }^{1}$ for the $Z_{c s}(3985)^{-}$follows naturally from the coupling structure of Eq. 2 . The possible meson clusters noted in Ref. 1 include the $\mathrm{D}^{0}, \mathrm{D}^{* 0}(2007)$, and $\mathrm{D}_{\mathrm{s}}^{-}$, with $\mathrm{J}^{\pi}$ values of $0^{-}, 1^{-}$, and $0^{-}$, respectively ${ }^{11}$. Although Ref. 11 does not assign a spin and parity value to the $D_{s}{ }^{*-}$, it must have a value of $1^{-}$ to meet the $1^{+}$assignment of Ref. 1 within the coupling restrictions of Eq. 2 . This conclusion follows from the coupling structure specified in Refs. 2-7. Accordingly, the $D_{s}{ }^{*-}$ is assumed to have a $J^{\pi}=1^{-}$assignment in this paper. Following Eq. 2, the $Z_{C S}(3985)^{-}$state has a $\mathbf{0}^{-} \times \mathbf{x} \mathbf{0} \times \mathbf{1}^{-}$structure that supports a J $\mathrm{J}^{\pi}=1^{+}$assignment in agreement with Ref. 1.

The first-order mass formula used in this paper partitions the tetraquark into two meson clusters. The first cluster is a scalar meson (sm) (e.g., $\mathrm{D}^{0}$ or $\mathrm{D}_{\mathrm{s}}{ }^{-}$) and the remaining cluster is a vector meson (vm) (e. g.,

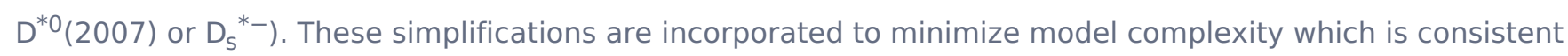
with an initial first-order formulation. In addition, the general tetraquark mass formula is assumed to have the form 2,3

$M=M_{s m}+M_{v m}+\Phi$

where $\boldsymbol{\Phi}$ defines the interaction between the meson clusters. Within the scope of this mass formula, the meson-meson cluster interaction is assumed to be weak and sufficiently small to be ignored. Accordingly, Eq. 3 
represents a quasimolecular four quark system whose basic character is a weakly bound meson-meson system.

\section{1 $\mathrm{Ds}^{-}+\mathrm{D}^{* 0}(2007)$ Meson Clusters}

The BESIII Collaboration noted two possible configurations for the $Z_{C S}(3985)^{-}$tetraquark. Section 3.1 addresses the $D_{S}{ }^{-}+D^{* 0}(2007)$ meson cluster and Section 3.2 described the $D_{S}{ }^{*-}+D^{0}$ configuration.

In the $D_{s}{ }^{-}+D^{* 0}(2007)$ configuration, the scalar meson is $D_{s}{ }^{-}$and the vector meson is $D^{* 0}(2007)$. For the $Z_{C S}(3985)^{-}$, the vector meson is the $D^{* 0}(2007)$ and not the $D^{0}$ ground state. Since the first-order $Z_{C S}(3985)^{-}$ tetraquark mass formula involves an excited $D^{* 0}(2007)$ meson cluster, the mass formula is modified to account for this excitation energy 2,3 :

$M=M_{s m}+M_{v m}^{*}+\Phi$

where

$M_{\mathrm{vm}}{ }^{*}=M_{\mathrm{vm}}+\Delta\left(D^{* 0}(2007)-D^{0}\right)$

and $\Delta$ is the $D^{* 0}(2007)-D^{0}$ energy difference $\left(142.02 \mathrm{MeV} / \mathrm{c}^{2}\right)^{11}$.

\section{2 $\mathrm{Ds}_{\mathrm{s}}^{{ }^{-}+}+\mathrm{D}^{0}$ Meson Clusters}

In the $D_{s}{ }^{*-}+D^{0}$ configuration, the scalar meson is $D^{0}$ and the vector meson is $D_{s}{ }^{*-}$. For the $Z_{C S}(3985)^{-}$, the vector meson is the $D_{s}{ }^{*-}$ with a mass of $2112.2 \mathrm{MeV} / \mathrm{c}^{2}$ and not the $\mathrm{D}_{\mathrm{s}}{ }^{-}$ground state with a mass of 1968.34 $\mathrm{MeV} / \mathrm{C}^{2}$. Since the first-order $Z_{\mathrm{CS}}(3985)^{-}$tetraquark mass formula involves an excited $\mathrm{D}_{\mathrm{s}}{ }^{*-}$ meson cluster, the mass formula is modified to account for this excitation energy 2,3 using Eq. 4. In this case, the vector meson mass is given by

$\mathbf{M}_{\mathrm{vm}}{ }^{*}=\mathbf{M}_{\mathrm{vm}}+\Delta\left(D_{\mathrm{s}}^{*-}-D_{\mathrm{s}}{ }^{-}\right)$

and $\Delta$ is the $D_{s}{ }^{*-}-D_{s}{ }^{-}$energy difference $\left(143.86 \mathrm{MeV} / \mathrm{c}^{2}\right)^{11}$.

\subsection{Results and Discussion}

The angular momentum coupling from Eq. 2 and the first-order mass formula of Eqs. 1 and 3-6 are used to construct a $Z_{\mathrm{cs}}(3985)^{-}$state. This state is modeled as noted in Sections 3.1 and 3.2.

The first-order mass formula provides a reasonable representation of the $Z_{c s}(3985)^{-}$for both configurations suggested in Ref. 1 (i.e., $D_{s}{ }^{-}+D^{* 0}(2007)$ and $D_{s}{ }^{*-}+D^{0}$ tetraquark structures). The $D_{s}{ }^{-}+D^{* 0}(2007)$ tetraquark structure results in a mass of $4107.8 \mathrm{MeV} / \mathrm{c}^{2}$ that is that is about 3.1\% larger than the experimental value. Somewhat better results are obtained with the $D_{s}{ }^{*-}+D^{0}$ tetraquark structure. This configuration results in a mass of $4068.0 \mathrm{MeV} / \mathrm{c}^{2}$ that is about $2.1 \%$ larger than the experimental value. Both results are in reasonable agreement with the measured value. The first order mass formula is not sufficiently accurate to select the correct configuration. It is possible that the physical state is a combination of these possible 
configurations $^{1}$.

Although these results are encouraging, they are based on a first-order mass formula with a number of uncertainties including the assumed quark masses ${ }^{10}$ and the magnitude of the meson-meson cluster interaction. However, the model does provide an initial description of the $Z_{C S}(3985)^{-}$and its $J^{\pi}=1^{+}$ assignment in terms of a tetraquark structure .

\subsection{Conclusions}

The first-order mass formula predicts two possible tetraquark configurations for the $Z_{c s}(3985)^{-}$. Both weakly bound $\mathrm{D}_{\mathrm{s}}{ }^{-}$(c-bar s) $+\mathrm{D}^{* 0}(2007)$ (c u-bar) and $\mathrm{D}_{\mathrm{s}}{ }^{*}$ - (c-bar s) $+\mathrm{D}^{0}$ (c u-bar) meson clusters lead to predicted mass values that are within about $3 \%$ of the measured value ${ }^{1}$. The predicted $\mathrm{J}^{\pi}=1^{+}$assignment is also in agreement with data ${ }^{1}$.

\section{References}

1) BESIII Collaboration, Observation of a Near-Threshold Structure in the K+ Recoil-Mass Spectra in e $+\mathrm{e}-\rightarrow$ $K+\left(D_{s}{ }^{-} D^{* 0}+D_{s}^{*}{ }^{*} D^{0}\right)$, Phys. Rev. Lett 126, 102001 (2021).

2) J. J. Bevelacqua, First-Order Tetraquark Mass Formula, Physics Essays 29, 198 (2016).

3) J. J. Bevelacqua, Description of the $X(5568)$ and Proposed $750 \mathrm{GeV} / \mathrm{c}^{2}$ State in Terms of a First-Order Tetraquark Mass Formula, Physics Essays 29, 367 (2016).

4) J. J. Bevelacqua, Fusion of doubly heavy mesons into a tetraquark, Physics Essays 31, 167 (2018).

5) J. J. Bevelacqua, Possible Tetraquark Explanation for the Proposed X(3872), Physics Essays 32, 469 (2019).

6) J. J. Bevelacqua, Description of the X(6900) as a Four Charmed Quark State in Terms of a First-Order Tetraquark Mass Formula, QEIOS KLXLKJ, 1 (2020).

https://doi.org/10.32388/KLXLKJ.

7) J. J. Bevelacqua, Description of the $X(2900)$ as an Open Flavor Tetraquark in Terms of a First-Order Mass Formula, QEIOS, OVLMEB, 1 (2020).

https://doi.org/10.32388/OVLMEB.

8) Ya. B. Zel'dovich and A. D. Sakharov, Kvarkovaia struktura i massy sil'novzaimodeistvuyushchikh chastits, Yad. Fiz. 4, 395 (1966).

9) A. D. Sakharov, Mass formula for mesons and baryons, Sov. Phys. JETP 51, 1059 (1980).

10) D. Griffiths, Introduction to Elementary Particles, $2^{\text {nd }}$ ed., (Wiley-VCH, Weinheim, 2008).

11) Particle Data Group, Review of Particle Physics, Prog. Theor. Exp. Phys. 2020, $083 C 01$ (2020). 